\title{
Local delivery of an antisense oligonucleotide for recessive dystrophic epidermolysis bullosa
}

R Mendes, L van Wissen, MW de Jager, F van der Ham, V Brinks, T Ritsema, EM Haisma | ProQR Therapeutics N.V. Leiden, the Netherlands

\section{Introduction}

Recessive dystrophic epidermolysis bullosa (RDEB) is a genetic blistering disorder caused by mutations in the COL7A1 gene encoding for type VII collagen (C7) protein. We have demonstrated that QR-313, a single stranded antisense oligonucleotide (AON), is efficient in the skipping of exon 73 from COL7A1 in human skin equivalents, thereby removing protein expression (figure 1$)^{12}$. One of the maing the restoration of C of potential medicines based AONs is sufficient delivery to the target or an. It has been shown that systemic (intravenous) administration eads to high AON concentrations in the liver and kidneys, with hardy detectable delivery to the skin. Hydrogels are part of the daily care of developed for QR-313.

\section{Aims}

In this study we wanted to assess Cy5-labeled QR-313 delivery in different (wounded) models, including human skin equivalents (HSEs), ex vivo human skin, ex vivo porcine skin and an in vivo wound model using minipigs. Cy5labeled QR-313 formulated into PBS or hydrogel was applied.

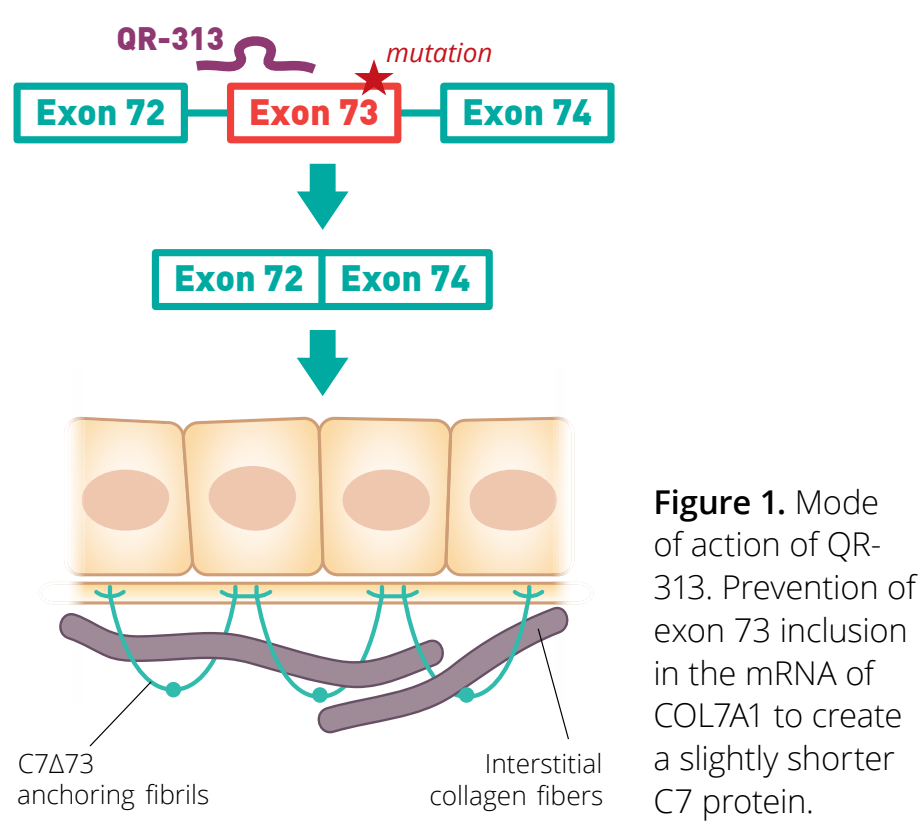

\section{Material and Methods}

ull thickness HSEs were grown from healthy skin donors in an arquid interface on filters. Ex vivo human skin was obtained, washed and excess dermis was removed manually, next the skin was prepped to fit onto filters to culture at the air-liquid interface. Ex vivo porcine skin was btained, the skin was prepped to $1 \mathrm{~mm}$ thickness using a dermatom and cultured at the air liquid interface. An in vivo minipig model was used, where $0.35 \mathrm{~mm}$ wounds where created using a dermatome. all models a DEB-like wound was created by removing the epidermis, was aith a scalpel or with a dermatome. Next Cy5-labeled QR-313 was applied onto the skin pieces in PBS or formulated in a hydroge. Cy5 diffusion into the skin models was followed for 24 hrs up to 7 days. All skin sections were processed for histology, stained with DAPI or hematoxylin and analyzed using (fluorescent) microscopy.

\section{Results}

1. Cy5-labeled QR-313 is delivered in wounded human skin equivalents

uman skin equivalents (HSES) were used to assess behavior of the AON in a 3D environment. Part of the epidermis was removed to mimic DBS wounds before application of Cy5-labeler 24 hours no penetration into the epidermis of intact models was observed. In the HSEs where part of the epidermis was removed diffusion into the dermal compartment of the model was observed. When Cy5-labeled QR-313 ermulated into the hydrogel more the epidermis was observed (figure 2).

Intact skin
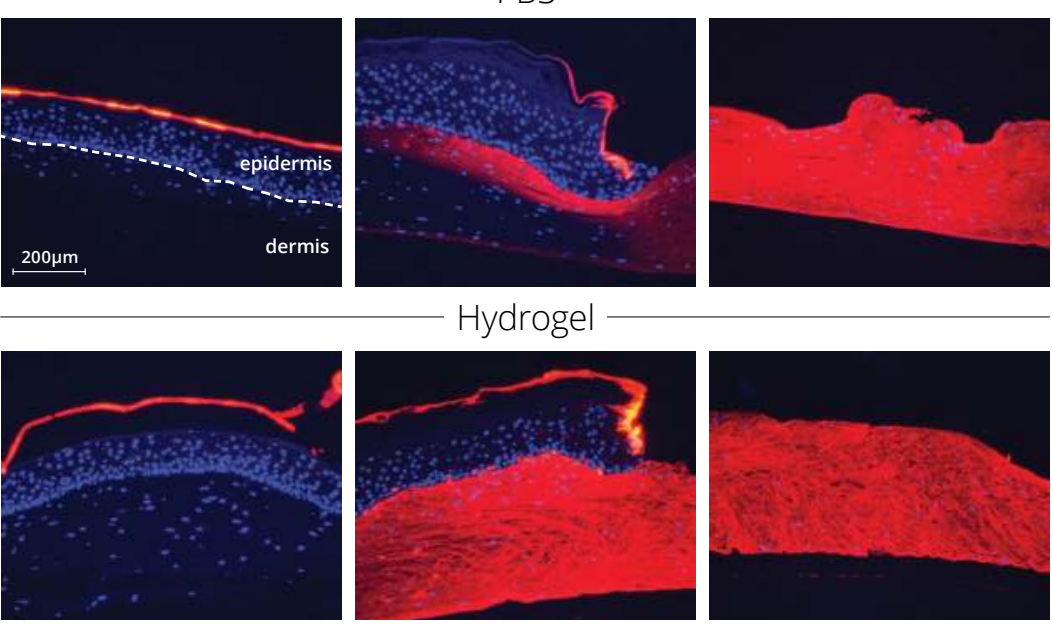

(avalents of Cy5-labeled QR-313 into two different formulations after $24 \mathrm{hrs}$. Top row shows application hydrogel. Cross sections of FFPE skin equivalents were counterstained with DAPI (blue) and the Cy5 QR-313 signal (red) was visualized by fluorescence microscopy.

\section{Results (continued)}

Next delivery of different concentrations of QR-313 in hydrogel were

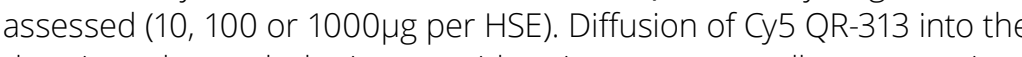
dermis underneath the intact epidermis was seen at all concentrations. The signal intensified with increasing concentrations of QR-313 in both. the wound bed and underneath the epidermal layer. This suggests more diffusion at higher QR-313 concentrations: in a dose-proportiona manner (figure 3).

Wound bed
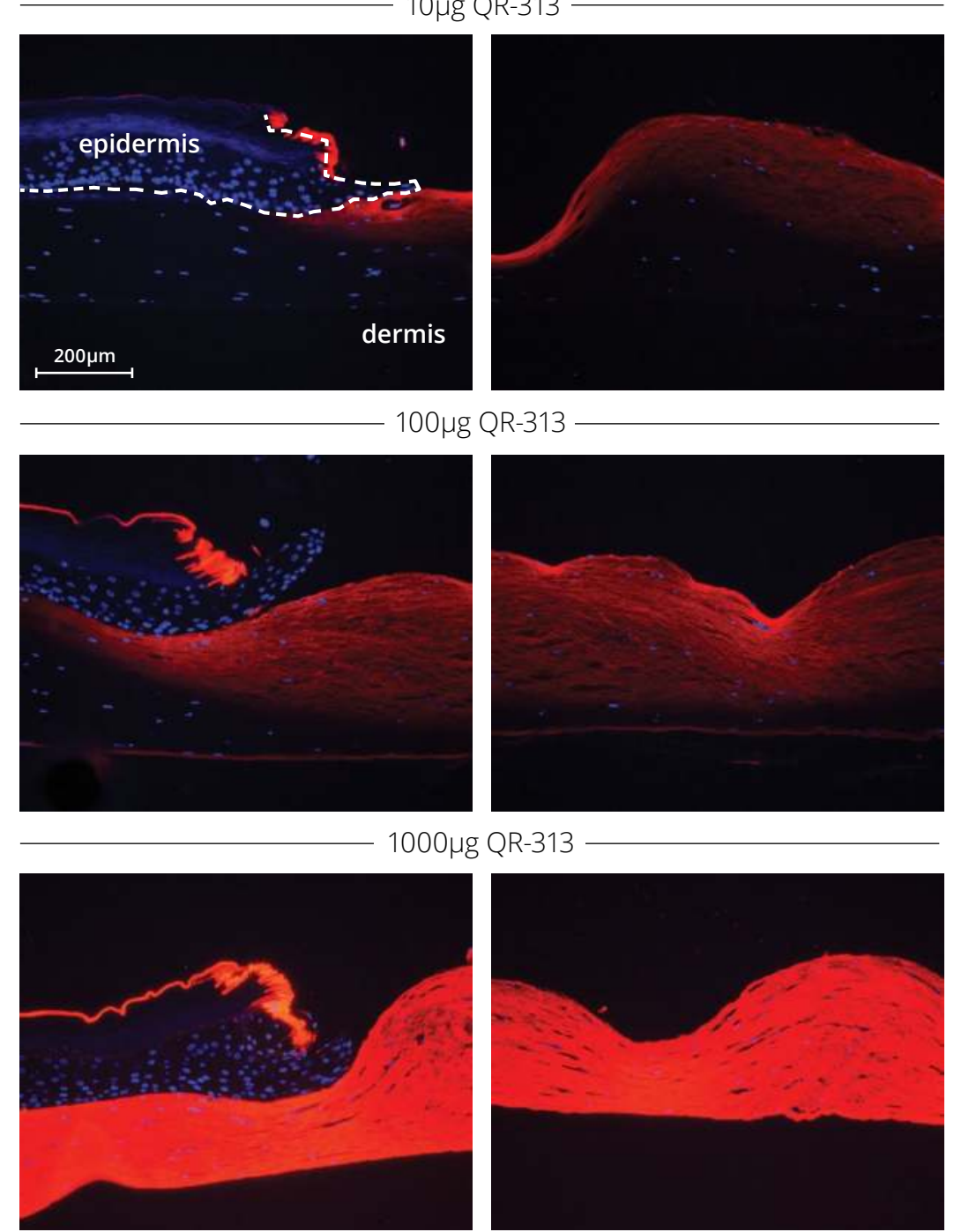

Figure 3. Delivery of different concentrations Cy5-labeled QR-313 formuled in hydrogel in HSEs. Three different concentrations were tested: 10, 100 or 1000 jg Cy5-labeled QR-313 was applied per HSE for $24 \mathrm{hrs}$. Cross sections of FFPE sections were counterstained with DAPI and the Cy5 signal was visualized by fluorescence microscopy.

2. Cy5-labeled QR-313 is delivered in the dermis of wounded ex vivo porcine and human skin

Application of Cy5-labeled QR-313 for 24 or 48 hrs onto intact and wounded ex vivo porcine skin demonstrated diffusion of Cy5-labeled QR-313 into the dermis, whereas in intact skin no Cy5-labeled QR313 was taken up. QR-313 formulated in hydrogel showed a deeper Similar resuls the dermis than QR-313 in solution (PBS) (figure 4) Similar results were obtained after application of Cy5-labeled QRhrs. Diffusian in the oligo was visible in skin where the epidermis was removed, almost no lateral diffusion was observed at the wound edges (figure 5).
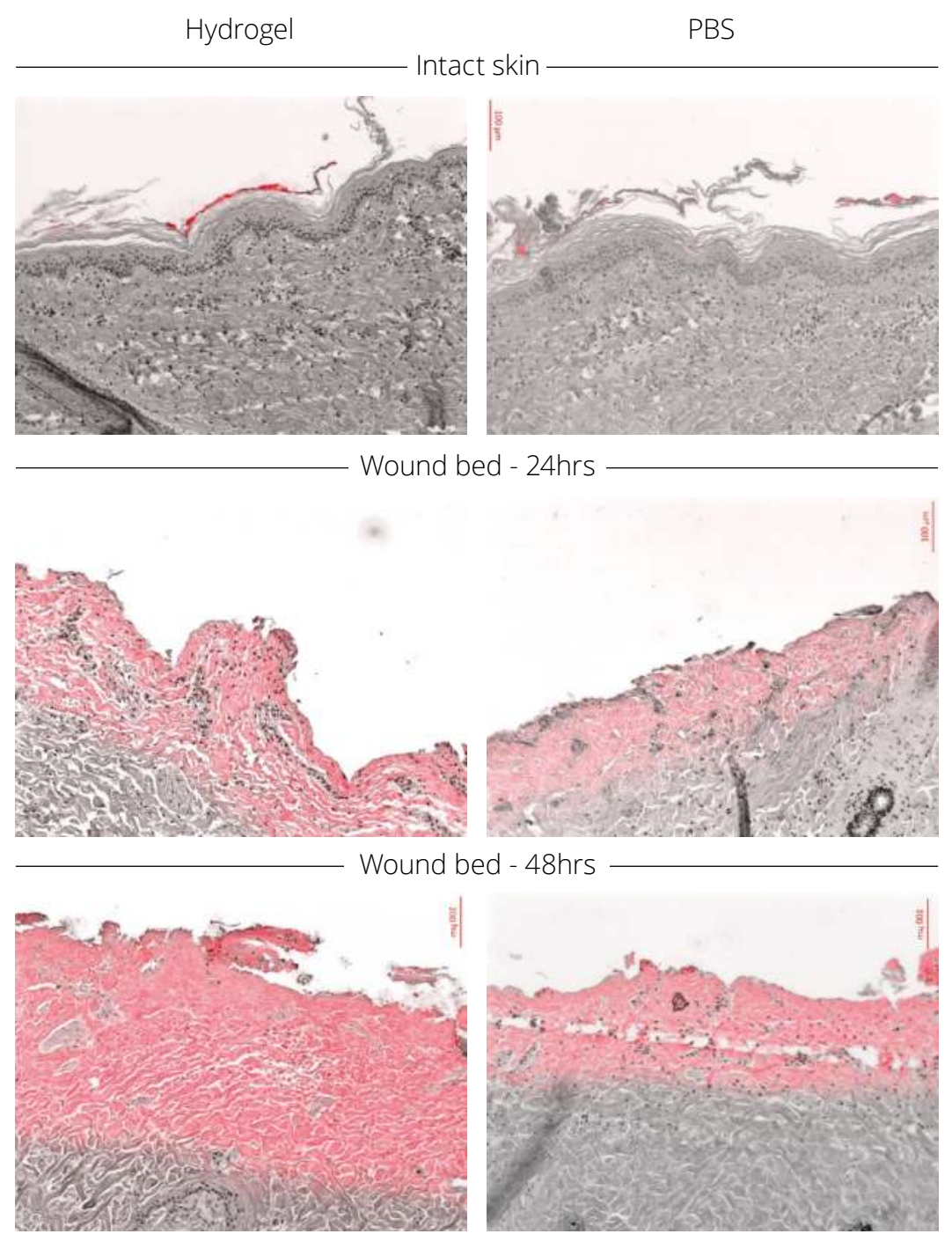

Figure 4. QR-313 delivery in ex vivo porcine skin of Cy5-labeled QR-313 hrs after application. Cy5-labeled QR-313 is indicated in red. Scale bar indicates $100 \mu \mathrm{m}$

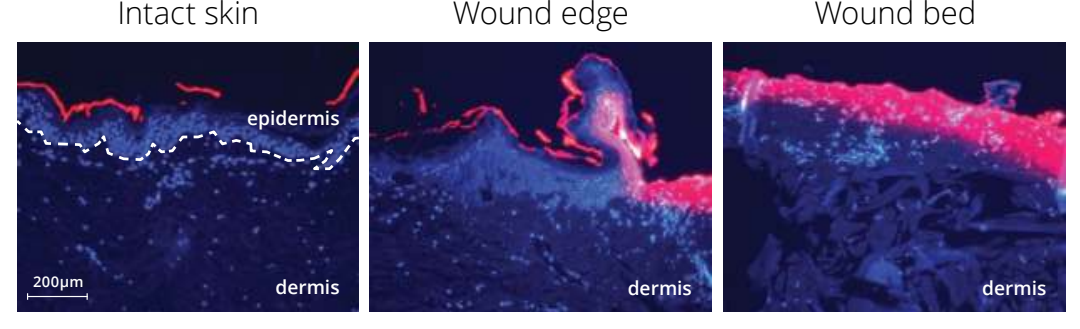

Figure 5. Delivery of Cy5-labeled QR-313 formulated into hydrogel counterstained with DAPI and the Cy5 QR-313 signal was visualized by

3. Cy5-labeled QR-313 is delivered in nuclei after application on wounded minipig skin

Wounds of $2 \times 3 \mathrm{~cm}$ and $0.35 \mathrm{~mm}$ deep were created onto the backs of minipigs. Delivery to the dermis was observed using Cy 5 QR-313 a strength of $0.5 \mathrm{mg} / \mathrm{g}(50 \mathrm{mg} / \mathrm{cm} 2)$ in hydrogel after 3 times dosing using a 48 hour dose interval. After 7 days the wounds were closed by a newly formed epidermis and the Cy5 signal was seen in the Confocal microscopy shows co-localization of the Cy5 label with the cell nuclei (figure 6). In agreement with the experiment with ex vivo models, no penetration of Cy5-labeled QR-313 was observed into intact skin.
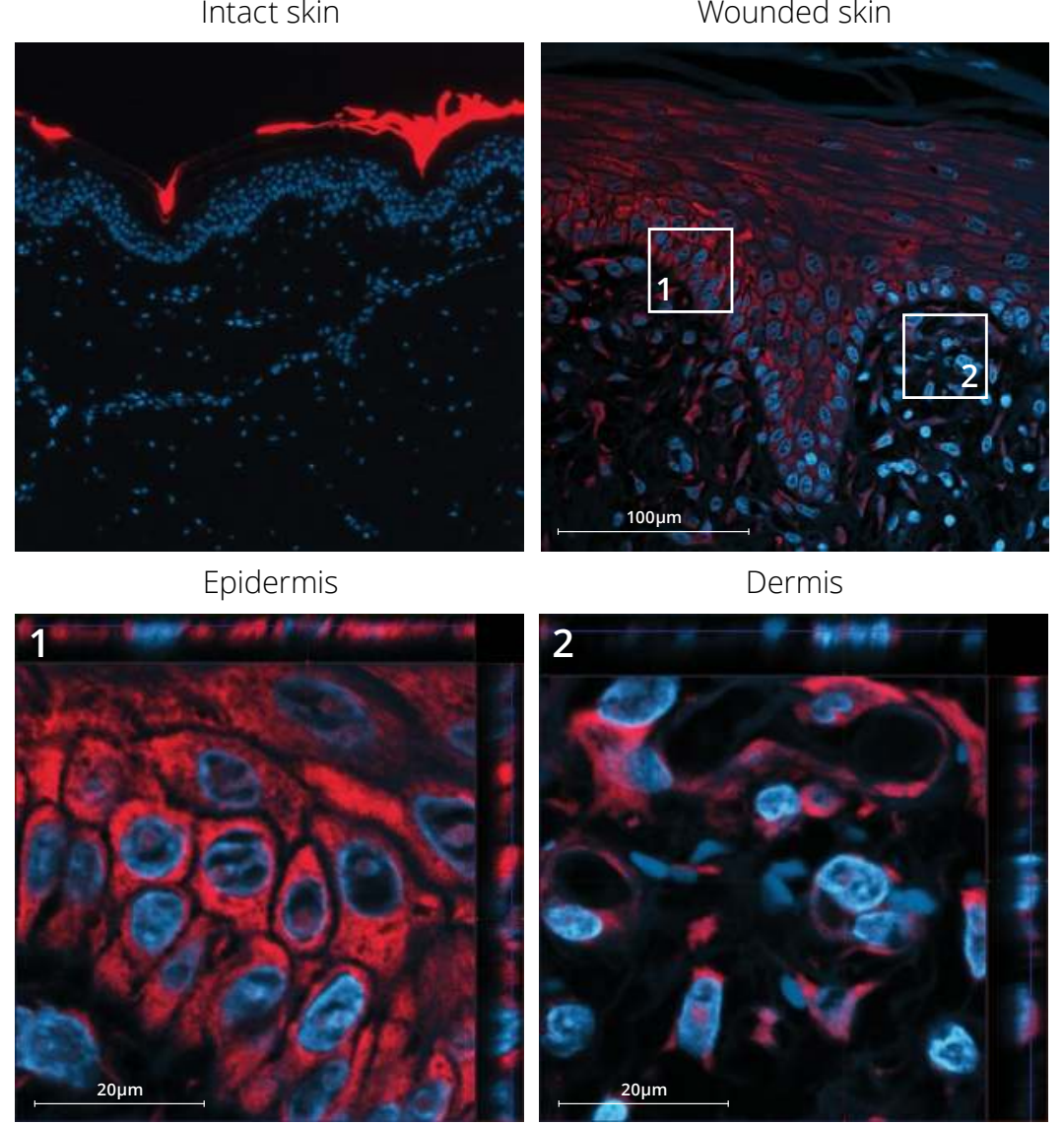

Figure 6. Cy5-labeled QR-313 delivery to wounded skin of minipigs. Cy5-labeled QR-313 was applied 3 times with 48 hrs intervals, 7 days fter wounding biopsies were were counterstained with Hoechst (blue) and the Cy5 signal (red) was Discussion

QR-313 is designed to exclude exon 73 from COL7A1 (see poster: In vitro evaluation of QR-313; an anti-sense oligonucleotide designed to skip exon 73 from the COL7A1 mRNA). In order to perform its function, QR-313 should be present in the nucleus. Based on the confocal images is efficiently delivery in minipig it is reasonable to

\section{Conclusion}

Here it is demonstrated that Cy5-labeled QR-313 delivery in vitro, ex vivo and in vivo models is successful in wounded skin, but not in intact skin. We conclude that hydrogel is a suitable formulation that enables diffusion of QR-313 into the skin of DEB patients at wound sites.

\section{Literature} Hovnanian, A. (2016). Targeted Exon Skipping Restores Type VII Collagen

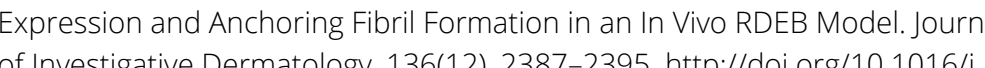
id.2016.07.029

列 prospect for dystrophic epidermolysis bullosa therapy. Molecular Therapy 